\title{
New Encoding Algorithm for Distributed Speech Recognition Based on DTFS Transform
}

\author{
Azzedine Touazi ${ }^{*}$ and Mohamed Debyeche \\ University of Sciences and Technology Houari Boumediene, El Alia 16111, Bab Ezzouar, \\ Algiers, Algeria \\ \{touazi.azzedine, mdebyeche\} @gmail.com
}

\begin{abstract}
The paper presents a new algorithm for efficient compression of front-end feature extracted parameters used in distributed speech recognition systems (DSR). In the proposed method the source encoder is mainly based on discrete time Fourier series (DTFS) by interpolation using Fourier coefficients with conventional vector quantization. The system provides a compression bit rate as low as $4 \mathrm{kbps}$; the experiments were carried out on the TIDigits Aurora2 database [1]. The simulation results show good recognition performance without dramatic change comparing with ETSI STQ-AURORA standard front-end feature compression algorithm with quantized features at $4.4 \mathrm{kbps}$ [2].
\end{abstract}

Keywords: Distributed speech recognition, Vector quantization, Discrete time Fourier series, Aurora2 database.

\section{Introduction}

The growth in wireless communication and mobile devices has supported the development of distributed speech recognition systems. Being developed and standardized by ETSI [2]. The basic idea of DSR consists of using a local Front end (FE) from which speech features are extracted and transmitted through a data channel to a remote Back end (BE) or remote server recognizer. The speech features used for recognition are the first 12 mel frequency cepstral coefficients (MFCCs) (c1-c12), the zeroth cepstral coefficient (c0) and the $\log$ energy $(\log \mathrm{E})$ in the frame. The 14dimentional feature vector is split into seven sub vectors; each of the sub vectors is encoded with a different 2-dim VQ.

The standard computes a feature vector every $10 \mathrm{~ms}$ and allocates 44 bits to each vector to achieve a total bit rate of $4400 \mathrm{bps}$ [2]. The number of bits allocated to each sub vector is shown in Table 1; with 8 bits are allocated to the (c0-log E) sub vector and 6 bits are allocated to the rest of each sub vector.

The Aurora 2 database consists of connected digit sequences for American English Talkers. It provides speech samples and scripts to perform speaker independent speech recognition experiments in clean and noisy conditions. This database has been prepared by down sampling to $8 \mathrm{kHz}$, filtering with the $\mathrm{G} .712$ characteristic; noise is

* Azzedine Touazi, Faculty of Electronic and Computer Sciences, Signal and Communication Laboratory (LCPTS). 
Table 1. Bits allocation used by Aurora

\begin{tabular}{llllllll}
\hline Sub-vector & $\mathrm{c} 1, \mathrm{c} 2$ & $\mathrm{c} 3, \mathrm{c} 4$ & $\mathrm{c} 5, \mathrm{c} 6$ & $\mathrm{c} 7, \mathrm{c} 8$ & $\mathrm{c} 9, \mathrm{c} 10$ & $\mathrm{c} 11, \mathrm{c} 12$ & $\mathrm{c} 0, \log \mathrm{E}$ \\
\hline Bits allocated & 6 & 6 & 6 & 6 & 6 & 6 & 8 \\
\hline
\end{tabular}

artificially added to the filtered TIDigits at a desired SNR (clean, 20, 15, 10, 5, 0, $5 \mathrm{~dB}$ ) and eight different noise conditions - Subway - Babble - Car - Exhibition hall Restaurant - Street - Airport - Train station. Furthermore, a full description of the Aurora2 database is given in [1].

Various schemes for compressing the MFCC vectors have been proposed in the literature. Among these methods are the coding based on discrete cosine transforms (DCT) [3] [4], another method that uses the predictive vector quantization [5]. Also, by analysis the statistical properties of the MFCC vectors a series of quantization schemes have been described in [6].

In this paper we have derived an interpolation method which operates in discrete time Fourier series domain (DTFS). This transform is widely used in signal processing such as spectral analysis and filter design.

In the proposed algorithm we exploit the temporal correlation characteristic between consecutive MFCC vectors extracted at regular period and transformed into DTFS domain, suggests that we do not have to transmit every spectral component (magnitudes and phases) of MFCC vector to the decoder; instead, we could transmit only one part of the spectral component at regular interval. However, at the decoder, the non transmitted spectral component could then be derived by means of linear interpolation from the adjacent components.

\section{Overall Description of the Algorithm}

At the client side, speech is first segmented into frames; features are computed for each frame of $10 \mathrm{~ms}$. As shown in figure 1 a normalized DTFS is performed and converted to the polar form with $N=14(\mathrm{c} 1, \ldots, \mathrm{c} 12, \mathrm{c} 0, \log \mathrm{E})$ and $k=0, \ldots, N / 2$, where the phase spectrum is discarded according to the following equations [7] [8] and [9]:

$$
S_{k}=\sqrt{A_{k}^{2}+B_{k}^{2}}, \quad \Phi_{k}=\arctan \left[B_{k} / A_{k}\right]
$$

With:

$$
\left.\left.\begin{array}{rl}
A_{k} & =\frac{2}{N} \sum_{n=0}^{N-1} s(n) \cos \left(\frac{2 \pi k n}{N}\right) \\
B_{k} & =\frac{2}{N} \sum_{n=0}^{N-1} s(n) \sin \left(\frac{2 \pi k n}{N}\right)
\end{array}\right\} \begin{array}{rl}
A_{k} & =\frac{1}{N} \sum_{n=0}^{N-1} s(n) \cos \left(\frac{2 \pi k n}{N}\right) \\
k=1,2, \ldots, N / 2-1 & \text { and } \quad \\
B_{k}=\frac{1}{N} \sum_{n=0}^{N-1} s(n) \sin \left(\frac{2 \pi k n}{N}\right)
\end{array}\right\} k=0, N / 2
$$

The polar components are $S=\left(s_{0}, s_{1}, \ldots, s_{7}\right)$ and $\Phi=\left(\varphi_{0}, \varphi_{1}, \ldots, \varphi_{7}\right)$. Since the equation (2), for $k=0$, we can demonstrate that $\varphi_{0}=0$. For the set of two successive feature vectors $\mathrm{MFCC}_{\mathrm{n}}$ and $\mathrm{MFCC}_{\mathrm{n}+1}$ we transmit both phase and amplitude spectra for $\mathrm{MFCC}_{\mathrm{n}}$ and just the phase spectrum for $\mathrm{MFCC}_{\mathrm{n}+1}$. 


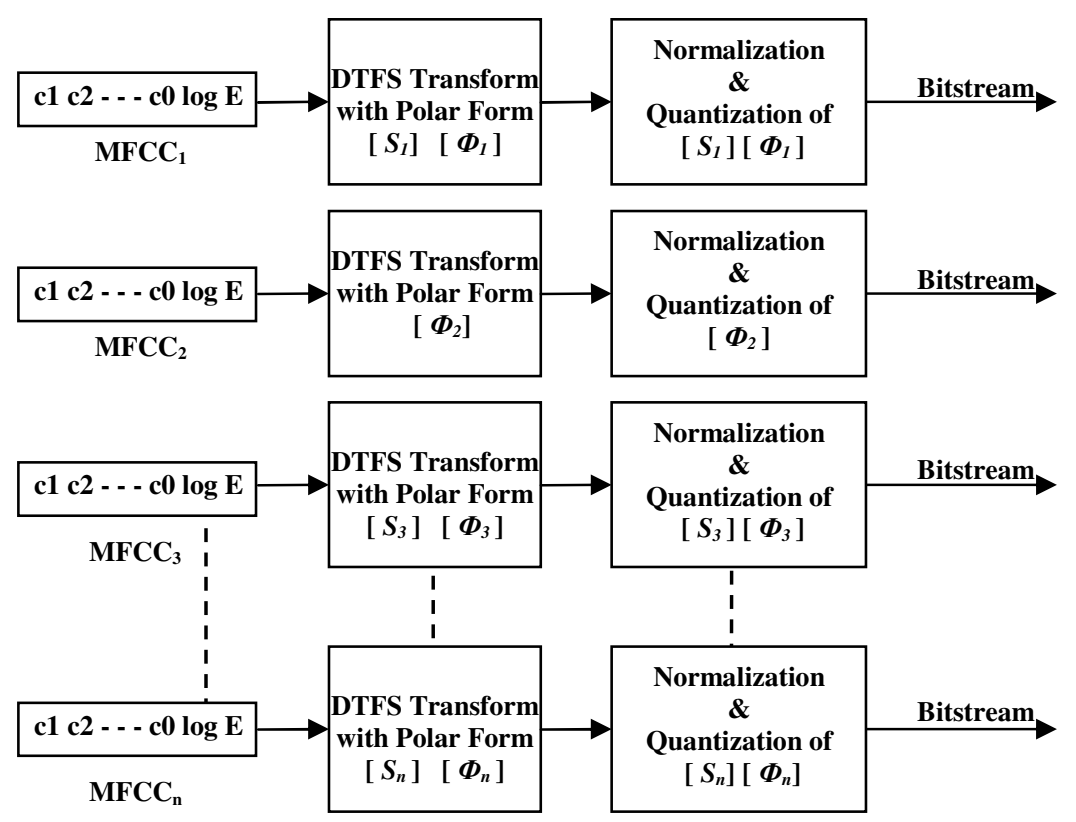

Fig. 1. DTFS transforming and quantization block

The choice to transmit the phase instead of the amplitude in the case of $\mathrm{MFCC}_{\mathrm{n}+1}$ is approved by an experiment with comparing the SNRs average (sets A, B and C) for interpolated DTFS components without quantization; as shown in figure below for the following cases: 1) Both amplitude and phase interpolation. 2) Amplitude interpolation. 3) Phase interpolation.

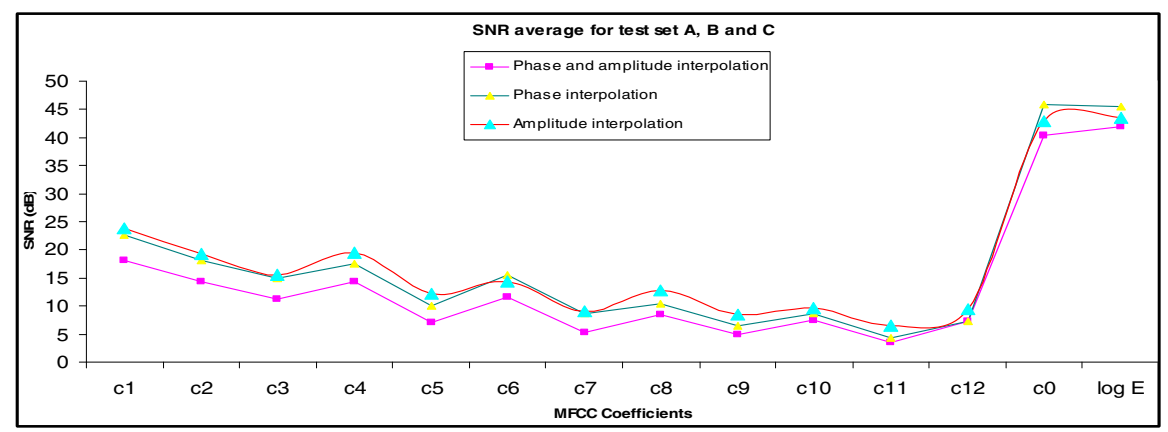

Fig. 2. SNR measurements average without quantization 
The SNRs average for the case of amplitude interpolation shows a greatest value comparing with the two other cases. We have also compared the SNRs average between interpolated amplitude spectra and Aurora encoder [2]. It can be seen from figure 3 that the SNR degrees in the case of amplitude interpolation are higher than the Aurora encoder for the first four coefficients (c1-c4) and are decreasing from the coefficient $\mathrm{c} 5$; for $(\mathrm{c} 0, \log \mathrm{E})$ the values are smoothly increased comparing with $\mathrm{Au}$ rora encoder. As it is well known that the lower feature coefficients provide the greatest contribution to the recognition performance [10]; therefore a DTFS transform with amplitude interpolation can lead to a minor influence in the recognition performance.

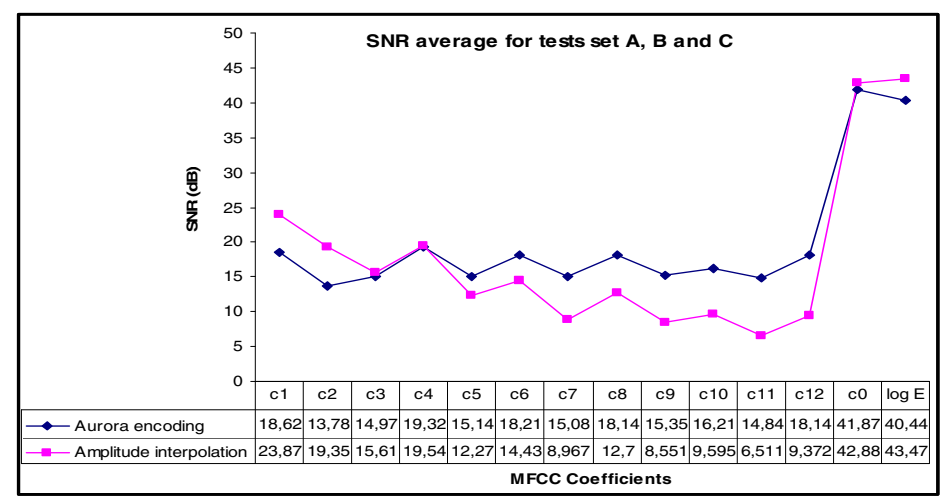

Fig. 3. SNR measurements average comparing with aurora encoder

In the quantization step, for two successive MFCC vectors the amplitude spectrum vectors $\left(s_{0}, s_{1}, \ldots, s_{7}\right)$ are encoded using split vector quantizer SVQ with the same codebooks, in which each vector is split equally into four sub-vectors and each one is quantized using its own VQ codebook trained with LBG algorithm [11] with codebooks of size 128 each.

The phase spectrum $\left(\varphi_{1}, \ldots, \varphi_{7}\right)$ is encoded using SVQ quantizer in which the vector is split into three sub vectors of ranks 2, 2 and 3 respectively, with codebooks of size 256 each in the case of $3.8 \mathrm{kbps}$ and 512 each in the case of $4.1 \mathrm{kbps}$.

The reason to choose more bits for encoding the phase of Fourier transform coefficients than encoding of spectral amplitude it's proved in reference [12]. The Table 2 shows the bits allocation for both cases of 3.8 and $4.1 \mathrm{kbps}$.

The decoding process at the back end consists of the inverse operations of the encoding in reverse order. However, the non-transmitted spectral component could then be derived by means of linear interpolation from adjacent components by:

$$
S_{M F C C_{n}}=\frac{S_{M F C C_{n-1}}+S_{M F C C_{n+1}}}{2}
$$


Table 2. Bits allocation at $3.8 \& 4.1 \mathrm{kbps}$

\begin{tabular}{llllll}
\hline & & \multicolumn{2}{l}{ Bits allocation at 3.8 kpbs } & \multicolumn{2}{l}{ Bits allocation at 4.1 kpbs } \\
\hline Polar form & Sub-vector & $\mathrm{MFCC}_{\mathrm{n}}$ & $\mathrm{MFCC}_{\mathrm{n}+1}$ & MFCC $_{\mathrm{n}}$ & MFCC $_{\mathrm{n}+1}$ \\
\hline \multirow{3}{*}{ Phase } & $(\varphi 1, \varphi 2)$ & 8 & 8 & 9 & 9 \\
& $(\varphi 3, \varphi 4)$ & 8 & 8 & 9 & 9 \\
& $(\varphi 5, \varphi 6, \varphi 7)$ & 8 & 8 & 9 & 9 \\
\multirow{5}{*}{ Amplitude } & $(s 0, s 1)$ & 7 & - & 7 & - \\
& $(s 2, s 3)$ & 7 & - & 7 & - \\
& $(s 4, s 5)$ & 7 & - & 7 & - \\
& $(s 6, s 7)$ & 7 & - & 7 & - \\
\hline
\end{tabular}
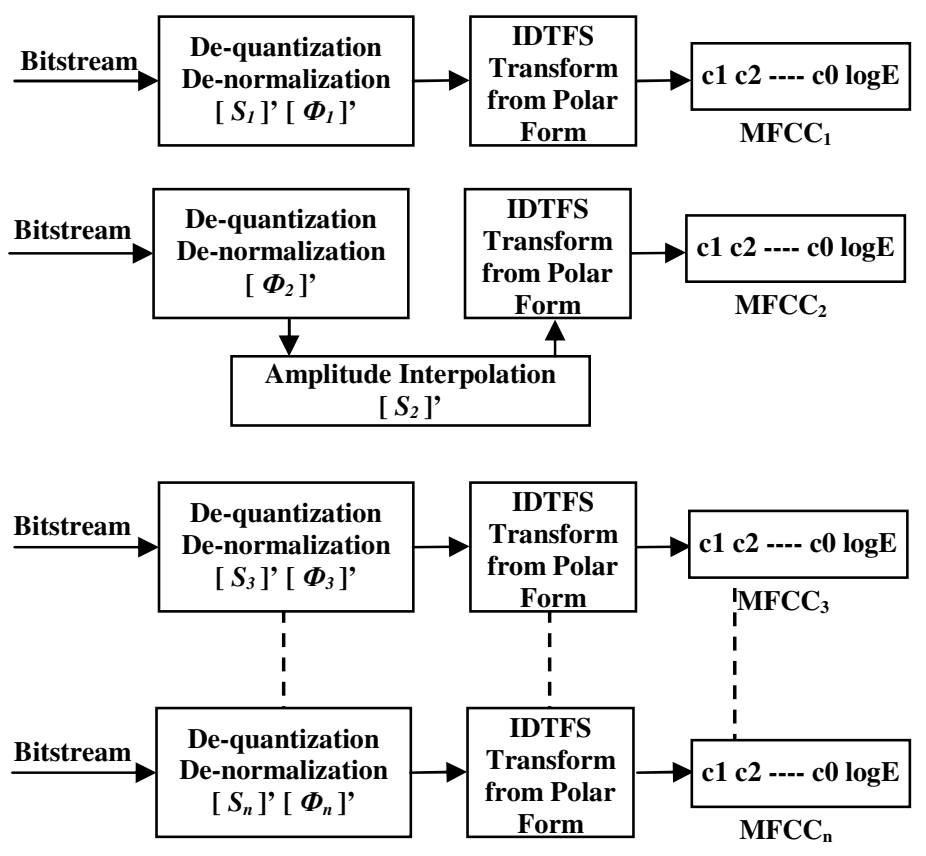

Fig. 4. DTFS de-quantization and interpolation block

\section{Recognition Results}

The experiments were carried out on the TIDigits Aurora corpus (sets A, B and C) with MFCCs extracted using the Aurora2 front-end [2] for both multi-condition and clean trainings. In the figure 5 we compared the SNR results for the following cases:

- Aurora encoder working at $4.4 \mathrm{kbps}$ [2].

- $\quad$ Proposed DTFS working at $3.8 \mathrm{kbps}$.

- $\quad$ Proposed DTFS working at $4.1 \mathrm{kbps}$. 


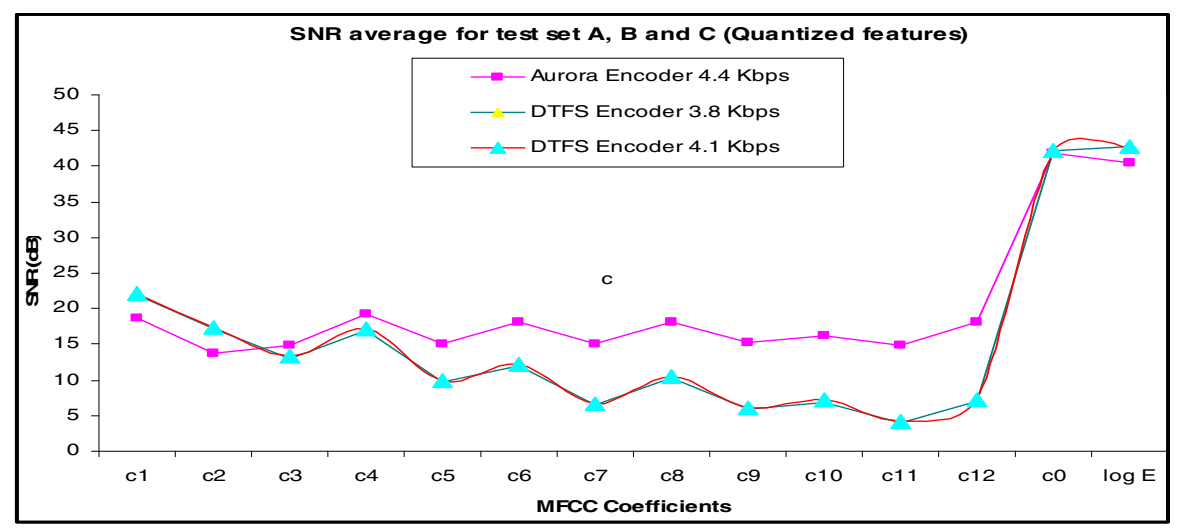

Fig. 5. SNR measurements average with quantization

It can be noticed a minor degradation from SNR levels after quantization; but for the first five MFCC coefficients we got an acceptable SNR values comparing with Aurora encoder. We note likewise when comparing (c0, log E).

The recognition were done using HTK 3.4 speech recognizer [13] to the coded MFCCs, while the $\mathrm{c} 0$ and $\log \mathrm{E}$ coefficients are both used in the recognition task; however, the results are compared for both compressed and uncompressed Aurora recognition.

In order to confirm our alternative the recognition accuracies average were firstly preformed on the uncompressed DTFS features, the Tables 3 and 4 show that transmitting DTFS coefficients with amplitude interpolation can lead to the good recognition performance.

Table 3. Recognition Accuracies in multi condition (uncompressed features)

\begin{tabular}{llll}
\hline & Test set A & Test set B & Test set C \\
\hline Aurora & 89.60 & 88.31 & 86.24 \\
Aurora encoding & 89.58 & 87.91 & 85.30 \\
Amplitude interpolation & 89.02 & 88.00 & 85.56 \\
Phase interpolation & 80.09 & 78.13 & 80.60 \\
Amplitude \& phase interpolation & 79.56 & 77.84 & 79.81 \\
\hline
\end{tabular}

Table 4. Recognition Accuracy in clean condition (uncompressed features)

\begin{tabular}{llll}
\hline & Test set A & Test set B & Test set C \\
\hline Aurora & 67.62 & 62.96 & 71.62 \\
Aurora encoding & 66.65 & 62.29 & 69.80 \\
Amplitude interpolation & 66.92 & 62.31 & 70.46 \\
Phase interpolation & 57.63 & 52.36 & 65.41 \\
Amplitude \& phase interpolation & 57.49 & 52.40 & 64.62 \\
\hline
\end{tabular}


We can see from Table below, in comparison with the compressed Aurora features, the proposed DTFS encoder with amplitude interpolation working at 3.8 and $4.1 \mathrm{kbps}$ maintains the same word level accuracies in the case of multi-condition, and the recognition accuracies are slightly inferior in the case of clean-condition.

Table 5. Word accuracy average (SNR: 0-20 dB), for test Sets A,B and C

\begin{tabular}{clcccc}
\hline Set & Training mode & \multicolumn{1}{c}{ Aurora standard Aurora at 4.4 kbps } & DTFS at 3.8 kbps & DTFS at 4.1 kbps \\
\hline \multirow{2}{*}{ A } & Clean & 67.62 & 66.65 & 65.70 & 65.70 \\
& Multi & 89.60 & 89.58 & 88.70 & 88.69 \\
\multirow{2}{*}{ B } & Clean & 62.96 & 62.29 & 61.07 & 61.15 \\
& Multi & 88.31 & 87.91 & 87.49 & 87.54 \\
\multirow{2}{*}{ C } & Clean & 71.62 & 69.80 & 68.90 & 69.17 \\
& Multi & 86.24 & 85.30 & 85.22 & 85.34 \\
\hline
\end{tabular}

The experiment results show that the proposed algorithm at low bit rates slightly affect the final speech recognition accuracy only by less than $1 \%$, so there is no significant difference in term of recognition accuracy.

\section{Conclusion}

The proposed algorithm working on DTFS domain focuses on reducing bit rate lower than $4 \mathrm{kbps}$; generally this encoder maintains the recognition performance without dramatic change comparing with Aurora encoder, with relatively more computational cost.

Also, the proposed technique can be extended to compress another kind of parameters that highly correlated with each other like LPC coefficients. Further work will involve improving both computational cost by proposing a new quantization techniques for the DTFS coefficients and recognition performance.

Acknowledgments. The authors would like to thank the LCPTS team, for providing contribution to carry out this research work.

\section{References}

1. Hirsch, H, G., D, Pearce.: The AURORA experimental framework for the performance evaluations of speech recognition systems under noisy conditions, [6th International Conference on Spoken Language Processing], ICSLP,China, October (2000)

2. ETSI Standard Document.: Speech Processing, Transmission and Quality Aspects (STQ); Distributed Speech Recognition; Front-end Feature Extraction algorithm Compression Algorithm, ETSI ES 201108 V 1.1.3, Sep. (2003)

3. Kiss, I., Kapanen, P.: Robust feature vector compression algorithm for distributed speech recognition. In Eurospeech (1999) 
4. Zhu, Q., Alwan, A.: An efficient and scalable 2D-DCT based feature coding scheme for remote speech recognition, in Proc. IEEE Int. Conf. Acoustic, Speech. Signal processing. (2001)

5. Jose Enrique, Garcia., Alfonso, Ortega., Antonio, Miguel., Eduardo, Lleida.: Predictive vector quantization using the M-algorithm for distributed speech recognition, VI Jornadas en Tecnología del Habla and II Iberian SLTech Workshop, FALA (2010)

6. So, Stephen., Kuldip, Paliwal, K.: Quantization of speech features, source coding, Automatic Speech Recognition on Mobile Devices and over Communication Networks Advances; http://www.springerlink.com/content/u1t465157615k202/, (2008)

7. Julius, O, Smith.: Mathematics of the discrete Fourier transform (DFT) with audio applications, Center for Computer Research in Music and Acoustics (CCRMA), Department of Music, Stanford University, https://ccrma.stanford.edu/ jos/mdft/; Viewed May (2011)

8. Eddie L, T, Choy.: Waveform Interpolation Speech Coder at $4 \mathrm{~kb} / \mathrm{s}$, Department of Electrical and Computer Engineering, McGill University Montreal, Canada August (1998)

9. Mohamed, Elfataoui., Gagan, Mirchandani.: A Frequency- Domain Method for Generation of Discrete-Time Analytic Signals., IEEE trans on signal processing, vol. 54, no. 9, september (2006)

10. Yongxin, Zhang.: Acoustic model and pronunciation adaptation in automatic speech recognition, University of Miami, (2006)

11. Linde, Y., Buzo, A., R.M, Gray.: An algorithm for vector quantizer design, IEEE Trans on Communications, Vol. 28, pp. 84-95, Jan. (1980)

12. Pearlman, W.A., Gray, R.M.: Source coding of the discrete Fourier transform. IEEE trans. on information theory. IT-24 (6): 683-692. (1978)

13. Young, S., Evermann, G., Gales, M., Hain, T., Kershaw, D., Liu, X., Moore, G., Odell, J., Ollason, D, Povey, D., Valtchev, V., Woodland, P.: The HTK Book, HTK Version 3.4, Cambridge University Engineering Department, (2006) 